\title{
Cognitive profile in patients with a first-ever lacunar infarct with and without silent lacunes: a comparative study
}

Lorena Blanco-Rojas ${ }^{1}$, Adrià Arboix ${ }^{1,2 *}$, David Canovas ${ }^{3,5}$, Marta Grau-Olivares ${ }^{1}$, Joan Carles Oliva Morera ${ }^{4}$ and Olga Parra 2,6

\begin{abstract}
Background: The detection of early neuropsychological abnormalities as precursors of cognitive decline of vascular origin in patients with lacunar stroke is a subject of increasing interest. The objective of this study was to assess whether there were differences in the performance of a battery of neuropsychological tests in first-ever lacunar stroke patients with and without associated silent multiple lacunar infarctions found incidentally on the brain magnetic resonance imaging (MRI) scan.

Methods: A total of 72 consecutive patients with first-ever lacunar infarction were studied 1 month after stroke. All patients underwent a comprehensive neuropsychological evaluation, which included the California Verbal Learning Test (CVLT), Phonetic Verbal Fluency Test (PMR), Semantic Verbal Fluency Test (category "animals"), Digit Span Forward and Backward from the Wechsler Adult Intelligence Scale (WAIS-III), and Mini-Mental State Examination (MMSE).

Results: A total of 38 patients (52.7\%) had silent multiple lacunar infarcts, with corona radiata as the most frequent topography $(P<0.023)$. White matter hyperintensities (leukoaraiosis) were observed in $81.1 \%$ of patients with silent multiple lacunar infarcts and in 50\% with a single lacunar infarction $(P<0.007)$. Patients in both groups showed similar scores in the MMSE, but those with associated silent lacunar infarctions showed a poorer performance in the semantic fluency test $(P<0.008)$ and in short delayed verbal memory $(P<0.001)$. In both cases, however, leukoaraiosis was not statistically significant in multivariate linear regression models adjusted by confounding covariates. In these models, multiple silent lacunar infarctions and education were independent predictors of poor performance in the semantic fluency test and in short delayed verbal memory.

Conclusions: The presence of silent multiple lacunar infarctions documented on brain MRI scans in patients with first-ever lacunar stroke was associated with mild neuropsychological abnormalities, particularly in the performance of executive functions (semantic fluency) and short delayed verbal memory. According to these findings, in the initial stages of small vessel disease, mild neuropsychological abnormalities appear to be related to lacunes rather than to leukoaraiosis or perivascular hyperintensities of vascular cause.
\end{abstract}

Keywords: California verbal learning test, Lacunar infarction, Neuropsychological abnormalities, MRI, Semantic fluency, Silent multiple lacunar infarctions, Vascular cognitive impairment

\footnotetext{
* Correspondence: aarboix@hscor.com

${ }^{1}$ Cerebrovascular Division, Department of Neurology, Capio-Hospital

Universitari del Sagrat Cor, Universitat de Barcelona, C/ Viladomat 288,

E-08029, Barcelona, Catalonia, Spain

${ }^{2}$ CIBER de Enfermedades Respiratorias (CB06/06), Instituto Carlos III, Madrid,

Spain

Full list of author information is available at the end of the article
} 


\section{Background}

Lacunar infarcts (LI) or lacunes account for $20-25 \%$ of all ischemic strokes [1,2]. According to recent studies, to distinct entities in patients with first-ever LI can be distinguished: 1) patients with a single LI in whom well known cardiovascular risk factors are present and 2) patients with multiple LI, in whom a high frequency of hypertension and leukoaraiosis have been found [3,4]. Also, mild neuropsychological abnormalities mainly executive dysfunction have been reported in $57 \%$ of patients with acute lacunar stroke [5] especially in patients with pure motor hemiparesis and atypical lacunar syndromes. In these circumstances, although general cognitive performance is usually normal, $55 \%$ of cases fulfill criteria of mild cognitive impairment of vascular type [6]. Identification of early stages of subcortical vascular dementia is an important area of research because this form of vascular dementia is one of the most common causes of cognitive decline in the elderly population $[7,8]$.

The potential impact of silent multiple lacunar infarctions on neuropsychological function in patients with clinically documented LI remains unclear. Therefore, the objective of this study was to assess whether there were differences in the performance of a battery of neuropsychological tests in first-ever lacunar stroke patients with and without associated silent multiple lacunar infarctions found incidentally on the brain magnetic resonance imaging (MRI) scan.

\section{Methods}

\section{Patients}

The study population consisted of 72 consecutive patients with first-ever LI admitted to the Department of Neurology of the Capio-Hospital Universitari Sagrat Cor, in Barcelona, Spain, between January 2006 and December 2011. All patients presented a lacunar syndrome according to the MillerFisher's classification [1]. Patients with cortical and/or subcortical non-lacunar infarct or intracerebral hemorrhage documented by MRI studies were excluded from the study as were those with severe cardiovascular disease, renal insufficiency, liver dysfunction, neoplastic or chronic disease, major depression and other psychiatric comorbidity (DSM-IV-R). Patients with impaired cognitive performance (Mini-Mental State Examination [MMSE] score $<24$ ) were also excluded.

The definitions of cerebrovascular risk factors and classic lacunar syndromes, including pure motor stroke, pure sensory stroke, sensorimotor stroke, ataxic hemiparesis, dysarthria-clumsy hand and atypical lacunar syndromes (patients presenting isolated dysarthria, dysarthria with facial paresis or isolated hemiataxia) were those used in previous studies [5,6,9]. Prior to conducting the study, approval was obtained from Ethical Committee on Clinical Research of the hospital. Written informed consent was obtained from all patients.

\section{MRI examination}

MRI studies were performed using a General Electric 1.5 Tesla Sigma system (General Electric, Milwaukee, WI) and the following sequences: T1-weighted $(\mathrm{TR}=479 \mathrm{~ms}$, $\mathrm{TE}=13 \mathrm{~ms}, \mathrm{FOV}=270 / 1.1$, slice thickness $5.0 \mathrm{~mm}$, GAP 1.0); $\mathrm{T} 2$-weighted ( $\mathrm{TR}=4885 \mathrm{~ms}, \mathrm{TE}=120 \mathrm{~ms}, \mathrm{FOV}=220$ / 1.1, slice thickness $5.0 \mathrm{~mm}$, GAP 2.0); fluid-attenuated inversion recovery (FLAIR) $(\mathrm{TR}=8000 \mathrm{~ms}, \mathrm{TE}=120 \mathrm{~ms}$, FOV $=240 / 1.1$, slice thickness $5.0 \mathrm{~mm}$, GAP 1.2); protonic density $(\mathrm{TR}=3400 \mathrm{~ms}, \mathrm{TE}=17 \mathrm{~ms}$, FOV $=240 / 1.1$, slice thickness $5.0 \mathrm{~mm}$, GAP 1.0); and diffusion sequences $(\mathrm{TR}=3350 \mathrm{~ms}, \mathrm{TE}=74 \mathrm{~ms}, \mathrm{FOV}=250 / 1.1$, slice thickness $5.0 \mathrm{~mm}$, GAP 1.0). Two senior radiologists determined the presence and topography of acute and chronic, single and/ or multiple silent lacunar infarcts by visual inspection of the T1, FLAIR, and T2 sequences of MRI scans. Patients were divided into two groups, those with a single LI and those with a single LI in association with multiple silent lacunar infarcts. Also, white matter hyperintensities (leukoaraiosis) were evaluated from T2-weighted, FLAIR and diffusion sequences and classified as present versus absent.

\section{Neuropsychological studies}

A battery of neuropsychological tests [10] was administered to all patients 1 month after the index admission. These included the California Verbal Learning Test (CVLT), Phonetic Verbal Fluency Test (PMR), Semantic Verbal Fluency Test (category "animals"), and Digit Span Forward and Backward from Wechsler Adult Intelligence Scale (WAIS-III). The Spanish versions of these instruments were used [11].

The CVLT is a standardized test for verbal learning and memory function developed to assess both the amount of material learned, recalled, and recognized, as well as qualitative aspects of how the verbal learning occurs or fails. A list of 16 words (List A) is presented five times. The words are equally drawn from four semantic categories with no consecutive words from the same category. Immediately after the fifth trial, a new list is read to the participant (List B) and asked to recall it. A short delayed recall test is presented immediately after recall of List $\mathrm{B}$, where the participant is asked to recall the words in List A. A long delayed recall test (CVLT-Long Delayed) is presented after a further 20 minutes interval. Finally, a "yes-no" recognition test consisting in the 16 items of List A, eight from List B and 20 random distracter items is presented [12]. The PMR test is used to assess semantic knowledge, retrieval ability and executive functioning. The patients have to say words beginning with letter "p" " $m$ " and " $r$ " in 60 seconds. In the Semantic Verbal Fluency Test (category "animals"), participants had to say as many words as possible from the semantic category "animals" in 60 seconds. The final measure is the total number of words. The Digit Span 
Forward and Backward Test is a subtest from WAIS-III, which is used to explore the short delayed verbal memory, working memory, and attention ability. The Digit Span Forward consists of serial numbers that the patient has to repeat in the same order than the evaluator. In the Backwards Test the patient has to say the serial numbers in the other way round.

\section{Statistical analysis}

Differences in demographics, vascular risk factors, clinical features, and results of neuropsychological tests between patients with single LI and those with associated multiple silent LIs were analyzed with the Student's $t$ test for continuous variables and the chi-square $\left(\chi^{2}\right)$ test or the Fisher's exact probability test (when appropriate) for categorical data. The degree of association of individual variables with multiple silent LIs was estimated by the odds ratio (OR) and the 95\% confidence interval (CI). Multiple linear regression analysis was performed to assess the effect of leukoaraiosis on neuropsychological performance adjusted by confounding covariates. Statistical significance was set at $P<0.05$.

\section{Results}

There were 36 men and 36 women, with a mean (standard deviation, SD) age of 75.4 (9.2) years. Thirty-four (47.2\%) patients presented a single LI and in the remaining 38 (52.8\%), associated silent multiple LIs were detected on the MRI scan. Table 1 shows the comparison of demographic variables, vascular risk factors, clinical features, and infarct topography between the groups of single and multiple silent LIs. Statistically significant

Table 1 Demographic, clinical and cognitive features of patients with single lacunar infarction (LI) and patients with associated multiple silent Lls

\begin{tabular}{|c|c|c|c|c|c|}
\hline \multirow[t]{3}{*}{ Variables } & \multicolumn{2}{|c|}{ Lacunar stroke patients } & \multirow[t]{3}{*}{ Odds ratio $(95 \% \mathrm{Cl})$} & \multirow[t]{3}{*}{$P$ value } & \multirow[t]{3}{*}{$t^{*}$} \\
\hline & Single LI & Multiple silent & & & \\
\hline & $(n=34)$ & Lls $(n=38)$ & & & \\
\hline Age, years, mean (SD) & $73.1(11.3)$ & $77.3(7.7)$ & & 0.999 & -1.834 \\
\hline Women, no. (\%) & $17(50)$ & $19(50)$ & & 0.999 & 0.000 \\
\hline Education, mean $(S D)^{\dagger}$ & $8.9(3.7)$ & $8.4(2.9)$ & & 0.71 & 0.672 \\
\hline MMSE, score, mean (SD) & $27.8(2.3)$ & $28(2.0)$ & & 0.686 & -0.406 \\
\hline High BP > 140/90 mm Hg, no. (\%) & $23(67.6)$ & $29(76.3)$ & $1.541(0.546-4.347)$ & 0.414 & -0.812 \\
\hline Diabetes mellitus, no. (\%) & $10(29.4)$ & $13(34.2)$ & $1.248(0.461-3.381)$ & 0.663 & -0.430 \\
\hline Dyslipidemia, no, (\%) & $10(29.4)$ & $12(31.5 \%)$ & $1.108(0.405-3.029)$ & 0.842 & -0.170 \\
\hline Toxic habits, no. $(\%)^{\ddagger}$ & $14(41.2 \%)$ & $15(39.5 \%)$ & $1.073(0.418-2.756)$ & 0.883 & -0.372 \\
\hline Lacunar syndrome, no. (\%) & & & & 0.381 & 0.360 \\
\hline Pure motor hemiparesis & $9(26.5)$ & $10(26.3)$ & - & & \\
\hline Pure sensory syndrome & $8(23.5)$ & $10(26.3)$ & $1.125(0.308-4.104)$ & & \\
\hline Dysarthria-clumsy hand & $5(14.7)$ & $9(23.7)$ & $1.62(0.392-6.678)$ & & \\
\hline Sensorimotor syndrome & $4(11.8)$ & $1(2.6)$ & $0.225(0.021-2.404)$ & & \\
\hline Ataxic hemiparesis & $2(5.9)$ & 0 & & & \\
\hline Atypical lacunar syndrome & $6(17.6)$ & $8(21.1)$ & $1.2(0.298-4.816)$ & & \\
\hline Infarct lateralization, no. (\%) & & & & 0.153 & -0.538 \\
\hline Right & $15(44.2)$ & $17(44.7)$ & - & & \\
\hline Left & $18(52.9)$ & $15(39.5)$ & $0.735(0.277-1.950)$ & & \\
\hline Bilateral & $1(2.9)$ & $6(15.8)$ & $5.294(0.570-49.136)$ & & \\
\hline Infarct topography, no (\%) & & & & 0.014 & -0.883 \\
\hline Basal ganglia & $2(5.9)$ & $6(15.8)$ & 7.500 (1.039-54.118) & & \\
\hline Corona radiata & $6(17.6 \%)$ & $17(44.7 \%)$ & $7.083(1.601-31.331)$ & & \\
\hline Internal capsule & $10(29.4 \%)$ & $4(10.5 \%)$ & - & & \\
\hline Thalamus & $9(26.5 \%)$ & $9(23.7)$ & $2.500(0.567-11.011)$ & & \\
\hline Pons & $7(20.6 \%)$ & $2(5.3)$ & $0.714(0.101-5.035)$ & & \\
\hline Leukoaraiosis, no. (\%) & $17(50)$ & $30(81.1)$ & $4.286(1.481-12.4)$ & 0.007 & -2.886 \\
\hline
\end{tabular}

${ }^{*} t$ : test statistics; ${ }^{\dagger}$ years of formal education; ${ }^{\ddagger}$ smoking/alcohol consumption. Abbreviations: Cl confidence interval, MMSE Mini-Mental State Examination, BP blood pressure. 
differences $(P=0.014)$ were only found in LI topography, with higher percentages of multiple silent LIs in the basal ganglia and corona radiata, and the presence of leukoaraiosis, which was significantly more frequent in patients with multiple silent LIs $(P=0.007)$. The remaining variables including clinical types of LI were similarly distributed in the two study groups. The mean (SD) MMSE score was 27.8 (2.3) in the group of single LI and 28 (2) in the group of multiple silent LIs $(P=0.686)$.

The results of neuropsychological tests are shown in Table 2. The group of patients with multiple silent LIs showed a poorer performance in semantic fluency (mean difference 3.265, 95\% CI 0.891-5.638; $P=0.008)$ and short delayed verbal memory (mean difference 1.265, 95\% CI $0.514-2.015 ; P=0.001$ ) as compared with the group of patients with single LI. Differences in the results of other tests were not statistically significant. There were no differences in the performance of neurological tests between the different lacunar subtypes.

In the multiple linear regression analysis to assess independent variables associated with performance of semantic fluency and short delayed verbal memory, the presence of leukoaraiosis was not statistically significant. In both models, multiple silent LIs and education were the only statistically significant variables (Table 3 ). Age almost reached statistical significance $(P=0.062)$ in the semantic fluency model.

\section{Discussion}

The clinical relevance of symptomatic lacunar stroke is well established but little is known of the impact of silent lacunes incidentally found on MRI scans on cognitive function. Previous studies have shown that a first single LI may cause cognitive decline as suggested by impairment in neuropsychological performance [3-5]. The present results indicate that lacunar stroke patients with associated multiple silent LIs had significantly poorer performance in some neuropsychological tests such as semantic verbal fluency and short term memory. These findings are even more interesting considering that patients were evaluated shortly after the acute cerebrovascular event (1 month).

Our results contribute to justify the presence of two distinct phenotypic entities (in risk factors, neuroimaging and neuropsychological profile) in lacunar strokes: LI caused by one isolated symptomatic lacune and lacunar stroke caused by one symptomatic LI in association with multiple clinically silent lacunar infarctions [1-4]. Also, leukoaraiosis was significantly more frequent in patients with multiple silent LIs, although a statistically significant role as an independent predictor of performance of semantic verbal fluency and short term memory was not observed in the multivariate linear regression analysis. The underlying small vessel vasculopathy may be different in the two LI entities [3,13,14]. A diffuse arteriopathy of perforating arteries with hyaline deposition (lipohyalinosis) in the group of multiple silent LIs and localized small vessel microatheroma at the origin of the deep perforating arteries (in single LI group). Although speculative, distinguishing these two clinical LI entities may enable more appropriate therapy.

In the present study, the neuropsychological impairment in the group of patients with associated multiple silent LIs probably results from the interruption of prefrontal-subcortical loops by LIs and white matter lesions. Interruptions of dorsolateral prefrontal-subcortical

Table 2 Results of neuropsychological tests in patients with single lacunar infarction (LI) and patients with associated multiple silent LIs

\begin{tabular}{|c|c|c|c|c|c|}
\hline \multirow[t]{3}{*}{ Variables } & \multicolumn{2}{|c|}{ Lacunar stroke patients } & \multirow[t]{3}{*}{ Mean difference $(95 \% \mathrm{Cl})$} & \multirow[t]{3}{*}{$P$ value } & \multirow[t]{3}{*}{$t^{*}$} \\
\hline & Single LI & Multiple silent & & & \\
\hline & $(n=34)$ & LIs $(n=38)$ & & & \\
\hline Phonetic verbal fluency test (PMR) & $25.30(14.90)$ & $20.30(10.57)$ & $4.997(-1.282-11.277)$ & 0.111 & 1.617 \\
\hline Semantic fluency (animals) & $14.84(5.15)$ & $11.84(4.72)$ & $3.265(0.891-5.638)$ & 0.008 & 2.746 \\
\hline \multicolumn{6}{|l|}{ California verbal learning test (CVLT) } \\
\hline CVLT short-delay (trial 1) & $4.51(1.69)$ & $3.25(1.42)$ & $1.265(0.514-2.015)$ & 0.001 & 3.366 \\
\hline CVLT learning (trial 1-5) & $4.66(2.74)$ & $5.5(2.138)$ & $-0.833(-2.066-0.399)$ & 0.182 & -1.349 \\
\hline CVLT long delay cued recall & $5.96(3.05)$ & $5.08(3.25)$ & $0.896(-0.634-2.407)$ & 0.249 & 1.163 \\
\hline CVLT long delay recognition & $12.06(3.14)$ & $11.69(3.24)$ & $0.376(-1.170-1.902)$ & 0.636 & 0.476 \\
\hline CVLT total learning (sum trial 1-5) & $36(10.57)$ & $32.63(9.5)$ & $3.361(-1.470-8.192)$ & 0.170 & 1.389 \\
\hline \multicolumn{6}{|l|}{ WAIS-III digit span forward } \\
\hline Direct scores & $11.63(4.24)$ & $11.44(2.78)$ & $0.191(-1.555-1.939)$ & 0.823 & 0.224 \\
\hline Scaled scores & $11.12(3.37)$ & $11.88(2.61)$ & $-0.767(-2.210-0.675)$ & 0.292 & -1.062 \\
\hline
\end{tabular}

"t: test statistics; $\mathrm{Cl}$ : confidence interval.

Data expressed as mean (standard deviation, SD) unless otherwise stated. 


\begin{tabular}{|c|c|c|c|c|}
\hline Variable & Coefficient (ß) & Standard error & t-statistic & $P$ value \\
\hline \multicolumn{5}{|l|}{ Semantic fluency model ${ }^{*}$} \\
\hline Constant & 19.845 & 4.825 & 4.112 & 0.000 \\
\hline Multiple silent lacunar infarcts & -2.255 & 1.027 & -2.194 & 0.031 \\
\hline Leukoaraiosis & -1.312 & 1.180 & -1.112 & 0.270 \\
\hline Age & -0.109 & 0.057 & -1.896 & 0.062 \\
\hline Education & 0.677 & 0.166 & 4.073 & 0.0001 \\
\hline \multicolumn{5}{|c|}{ Short delay verbal memory model ${ }^{\dagger}$} \\
\hline Constant & 6.039 & 1.619 & 3.729 & 0.0004 \\
\hline Multiple silent lacunar infarcts & -0.996 & 0.344 & -2.889 & 0.005 \\
\hline Leukoaraiosis & -0.351 & 0.395 & -0.887 & 0.378 \\
\hline Age & -0.028 & 0.019 & -1.453 & 0.151 \\
\hline Education & 0.195 & 0.056 & 3.500 & 0.0008 \\
\hline
\end{tabular}

*Adjusted R-squared: 0.3903; $P$ value: 2.802 .

${ }^{\dagger}$ Adjusted R-squared: 0.3463; P value: 2.365 .

loop results in executive dysfunction [15,16]. Patients with multiple silent LIs showed a poorer cognitive performance related to short delayed verbal memory and frontal functions than patients with a single LI. For this reason, some studies have suggested that this type of silent lacunes had a significant individual contribution to the risk of cognitive impairment and motor speed and executive functions, while global cognitive functions and memory functions remained unaffected $[17,18]$. On the other hand, cognitive impairment associated with vascular disease frequently does not fulfill the traditional criteria for degenerative dementia, because the criteria of Alzheimer's disease requires the presence of prominent memory impairment, which in contrast is not the main sign of vascular cognitive impairment. Patients with vascular dementia show a better long-term memory and greater deficits in frontal executive functioning than patients with Alzheimer's disease [18]. The neuropsychological assessment reveals planning, organization and abstraction impairment, as well as deficits in category fluency initiation, reasoning, mental flexibility, sequencing, fine motor performance, or attentional allocation source [19-21].

It may be argued that multiple silent LIs may be associated with a higher risk of cognitive impairment in the mid- and long-term [22]. However, further studies are needed to assess differences in prognosis regarding cognitive function in patients with single LI versus patients with multiple silent LIs. Cognitive stimulation and physical activities could be recommended to improve affected cognitive functions. In the LADIS (Leukoaraiosis and Disability) study, physical activity reduced the risk of cognitive impairment, mainly vascular dementia, in older people living independently [23].
A limitation of the study is the fact that leukoaraiosis was assessed qualitatively as presence versus absence and not quantified using a quantitative measure (e.g. Scheltens scale, Wahlund scale, Fazekas scale). In a community sample of asymptomatic participants aged 50 to 65 years, it has been shown that only deep white matter hyperintensities, not periventricular hyperintensities were related to diminished cognitive function in middleaged individuals [24]. The LADIS study [25] has shown that white matter hyperintensity volume was the strongest predictor of cognitive decline in cerebral small vessel disease, but also incident lacunes on MRI parallel a steeper rate of decline in executive functions and psychomotor speed. Accordingly, in addition to white matter lesions, lacunes determine longitudinal cognitive impairment in small vessel disease. Although the individual contribution of lacunes on cognition was modest, they cannot be considered benign findings, but indicate a risk of progressive cognitive impairment. In the present study carried out in patients with first-ever lacunar stroke and without cognitive impairment (MMSE mean score of 27.8 and 28 in the groups of single LI and multiple silent LIs, respectively), early neuropsychological alterations could be initially related to lacunes. A subsequent increase in leukoaraiosis may cause more significant neuropsychological alterations of the type of subcortical cognitive impairment. Subcortical LIs have been identified as the cause of mild cortical atrophy [26] and, although this was not analyzed in the present study, may also influence on mild incipient neuropsychological alterations observed in our patients.

The fact that some patients were included in a clinical trial (SPS3 randomized trial) of secondary prevention of cerebral ischemia (aspirin $325 \mathrm{mg} /$ day versus aspirin 
$325 \mathrm{mg} /$ day plus clopidogrel $75 \mathrm{mg} /$ day) may be view as a limitation of the study. However, participation in the trial did not interfere with radioimaging and neuropsychological studies of the patients because all those included in the present study presented a first-ever lacunar stroke.

\section{Conclusions}

The presence of multiple silent LIs documented on brain MRI scans in patients with first-ever lacunar stroke 1 month after the acute cerebrovascular event was associated with mild neuropsychological abnormalities, particularly in the performance of executive functions (semantic fluency) and short delayed verbal memory. It has been shown that silent lacunes themselves exert an independent effect on cognitive function [27]. In the initial stages of small vessel disease, mild neuropsychological abnormalities appear to be related to lacunes rather than to leukoaraiosis or perivascular hyperintensities of vascular cause.

\section{Abbreviations}

Cl: Confidence interval; CVLT: California verbal learning test; LI: Lacunar infarction; MMSE: Mini mental state examination; MRI: Magnetic resonance imaging; OR: Odds ratio; SD: Standard deviation; SVD: Subcortical vascular dementia; WAIS-III: Wechsler adult intelligence scale version III.

\section{Competing interests}

The authors declare that they have no competing interests.

\section{Authors' contributions}

LB was the principal investigator, designed the study, collected the data, did the neuropsychological assessments, contributed to analyze the data, interpreted the results and wrote the paper. AA diagnosed and took care of the patients, participated in the study design, analysis and interpretation of data and wrote the part of the paper related to the neurological issues. He is also the corresponding author. DC participated in the collection of data, medical care of the patients and review of the manuscript for intellectual content. MGO contributed to write the paper, edited the manuscript and provide editorial assistance, including the selection of the journal. JCSM and OP participated in the collection of data, medical care of the patients and review of the manuscript for intellectual content. All authors have read and approved the final draft.

\section{Acknowledgments}

We thank Elisenda Grivé, MD, from the Neuroradiology Service for her assistance in the neuroimaging location of lacunar infarcts, Oscar Benavente, $M D$, and Ana Roldan, MD, for their contribution to the article with some patients included 'Secondary Prevention of Small Subcortical Strokes' (SPS3) trial, Maria Mataró, MD, from the University of Barcelona for valuable scientific commentaries and Marta Pulido, MD; for editing the manuscript and editorial assistance.

\section{Author details}

${ }^{1}$ Cerebrovascular Division, Department of Neurology, Capio-Hospital Universitari del Sagrat Cor, Universitat de Barcelona, C/ Viladomat 288, E-08029, Barcelona, Catalonia, Spain. ${ }^{2} \mathrm{CIBER}$ de Enfermedades Respiratorias (CB06/06), Instituto Carlos III, Madrid, Spain. ${ }^{3}$ Department of Neurology, Hospital Parc Taulí, Sabadell, Barcelona, Catalonia, Spain. ${ }^{4}$ Fundació Parc Taulí, Corporació Sanitària Universitària Parc Taulí, Universitat Autònoma de Barcelona, Sabadell, Barcelona, Catalonia, Spain. ${ }^{5}$ Universitat Autònoma de Barcelona, Cerdanyola del Vallès, Barcelona, Catalonia, Spain. ${ }^{6}$ Department of Pneumology, Capio-Hospital Universitari del Sagrat Cor, Universitat de Barcelona, Barcelona, Catalonia, Spain.
Received: 27 November 2012 Accepted: 11 December 2013

Published: 16 December 2013

\section{References}

1. Arboix A, Martí-Vilalta JL: Lacunar stroke. Expert Rev Neurother 2009, 9:179-196.

2. Fisher CM: Lacunar infarcts. A review. Cerebrovasc Dis 1991, 1:311-320.

3. Boiten J, Lodder J, Kessels F: Two clinically distinct lacunar infarct entities? A hypothesis. Stroke 1993, 24:652-656.

4. Arauz A, Murillo L, Cantú C, Barinagarrementería F, Higuera J: Prospective study of single and multiple lacunar infarcts using magnetic resonance imaging. Risk factors, recurrence, and outcome in 175 consecutive cases. Stroke 2003, 34:2453-2458.

5. Grau-Olivares M, Arboix A, Bartrés-Faz D, Junqué C: Neuropsychological abnormalities associated with lacunar infarction. J Neurol Sci 2007, 257:160-165.

6. Grau-Olivares M, Bartres-Faz D, Arboix A, Rovira M, Junqué C: Mild cognitive impairment after lacunar infarction: voxel-based morphometry and neuropsychological assessment. Cerebrovasc Dis 2007, 23:353-361.

7. Jacoba C, Perace LA, Costello R, McClure LA, Holliday SL, Hart RG, Benavente OR: Cognitive impairment in lacunar strokes: the SPS3 trial. Ann Neurol 2012, 72:351-362.

8. Grau-Olivares M, Arboix A, Junqué C, Arenaza-Urquijo EM, Rovira M, BartrésFaz D: Progressive gray matter atrophy in lacunar patients with mild cognitive impairment. Cerebrovasc Dis 2010, 30:157-166.

9. Arboix A, García-Eroles L, Massons J, Oliveres M, Targa C: Hemorrhagic lacunar stroke. Cerebrovasc Dis 2000, 10:229-234.

10. Lezak MD, Howieson DB, Loring DW: Neuropsychological assessment. 4th edition. New York: Oxford University Press; 2004.

11. Arteriola i Fortuny L, Hermisollo Romo D, Pardee RE: Manual de normas y procedimientos para la batería neuropsicológica en español (Handbook of norms and procederes for the neuropsychological battery in Spanish)[in Spanish. 3rd edition. Tucson: Arizona Press; 1999:33-34.

12. Delis DC, Kramer JH, Kaplan E, Ober BA: California Verbal Learning Test. San Antonio, TX: The Psychological Corporation; 1987.

13. Lee PH, Bang OY, Hwang EM, Lee JS, Joo US, Mook-Jung I, Huh K: Circulating beta amyloid protein is elevated in patients with acute ischemic stroke. J Neural Transm 2005, 112:1371-1379.

14. Gomis M, Sobrino T, Ois A, Millán M, Rodríguez-Campello A, Pérez de la Ossa N, Rodríquez- González R, Jiménez-Conde J, Cuadrado-Godia E, Roquer J, Dávalos A: Plasma $\beta$-amyloid ${ }_{1-40}$ is associated with the diffuse small vessel disease subtype. Stroke 2009, 40:3197-3201.

15. Roman GC, Erkinjuntti T, Wallin A, Pantoni L, Chui HC: Subcortical ischaemic vascular dementia. Lancet Neurol 2002, 1:426-436.

16. Frisoni GB, Galluzzi S, Bresciani L, Zanetti O, Geroldi C: Mild cognitive impairment with subcortical vascular features: clinical characteristics and outcome. J Neurol 2002, 249:1423-1432.

17. Erkinjuntti T, Pantoni L: Subcortical vascular dementia. In Yearbook of Alzheimer, S Disease and Related Disorders, Chapter IX. Edited by Gauthier S, Cummings JL. London: Marin Dunitz; 2000:101-133.

18. Mendez MF, Ashla-Mendez M: Differences between multi-infarct dementia and Alzheimer's disease on unstructured neuropsychological tasks. J Clin Exper Neuropsychol 1991, 13:923-932.

19. Almkvist O, Backman L, Basun H, Wahlund LO: Patterns of neuropsychological performance in Alzheimer's disease and vascular dementia. Cortex 1993, 29:661-673.

20. Padovani A, Di Piero V, Bragoni M, lacoboni M, Gualdi GF, Lenzi GL: Patterns of neuropsychological impairment in mild dementia: a comparison between Alzheimer's disease and multi-infarct dementia. Acta Neurol Scand 1995, 92:433-442.

21. Lafosse JM, Reed BR, Mungas D, Starling SB, Wahbeh H, Jagust WJ: Fluency and memory differences between ischemic vascular dementia and Alzheimer's disease. Neuropsychology 1997, 11:514-522.

22. Wen W, Sachdev P: The topography of white matter hyperintensities on brain MRI in healthy 60 to 64 year old individuals. Neuroimage 2004, 22:144-154

23. Verdelho A, Madureira S, Ferro JM, Baezner $H$, Blahak C, Poggesi A, Hennerici M, Pantoni L, Fazekas F, Scheltens P, Waldemar G, Wallin A, Erkinjuntti T, Inzitari D, LADIS Study: Physical activity prevents progression for cognitive impairment and vascular dementia: results from the LADIS (Leukoaraiosis and Disability) study. Stroke 2012, 43:3331-3335. doi: 10.1161/STROKEAHA.112.661793. 
24. Soriano-Raya JJ, Miralbell J, López-Cancio E, Bargalló N, Arenillas JF, Barrios M, Cáceres C, Toran P, Alzamora M, Dávalos A, Mataró M: Deep versus periventricular white matter lesions and cognitive function in a community sample of middle-aged participants. I Int Neuropsychol Soc 2012, 18:874-885.

25. Jokinen H, Gouw AA, Madureira S, Ylikoski R, van Straaten EC, van der Flier WM, Barkhof F, Scheltens P, Fazekas F, Schmidt R, Verdelho A, Ferro JM, Pantoni L, Inzitari D, Erkinjuntti T, LADIS Study Group: Incident lacunes influence cognitive decline: the LADIS study. Neurology 2011, 76:1872-1878. doi: 10.1212/WNL.0b013e31821d752f.

26. Smith EE, Arboix A: Focal cortical thinning is caused by remote subcortical infarcts: spooky action at a distance. Neurology 2012, 79:2016-2017.

27. Arboix A: Lacunar infarct and cognitive decline. Expert Rev Neurother 2011, 11:1251-1254.

doi:10.1186/1471-2377-13-203

Cite this article as: Blanco-Rojas et al:: Cognitive profile in patients with a first-ever lacunar infarct with and without silent lacunes: a comparative study. BMC Neurology 2013 13:203.

\section{Submit your next manuscript to BioMed Central and take full advantage of:}

- Convenient online submission

- Thorough peer review

- No space constraints or color figure charges

- Immediate publication on acceptance

- Inclusion in PubMed, CAS, Scopus and Google Scholar

- Research which is freely available for redistribution 